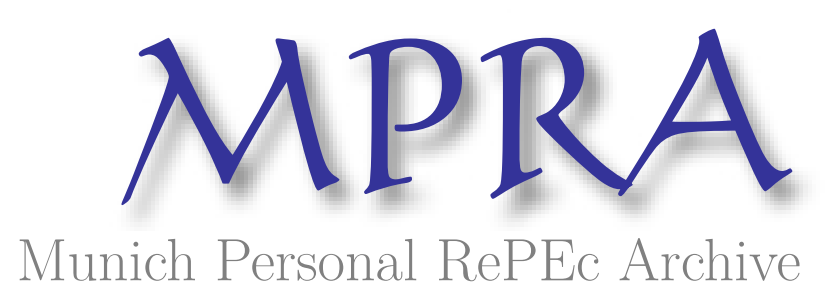

\title{
Optimal Portfolio Liquidation for CARA Investors
}

Schied, Alexander and Schöneborn, Torsten

27 September 2007

Online at https://mpra.ub.uni-muenchen.de/5075/

MPRA Paper No. 5075, posted 29 Sep 2007 UTC 


\title{
Optimal portfolio liquidation for CARA investors
}

\author{
Alexander Schied \\ Department of Mathematics \\ TU Berlin \\ Strasse des 17. Juni 136 \\ 10623 Berlin, Germany \\ schied@math.tu-berlin.de
}

\author{
Torsten Schöneborn \\ Quantitative Products Laboratory \\ Deutsche Bank \\ Alexanderstr. 5 \\ 10178 Berlin, Germany \\ schoeneborn@math.tu-berlin.de
}

Version: September 27, 2007

\begin{abstract}
We consider the finite-time optimal portfolio liquidation problem for a von Neumann-Morgenstern investor with constant absolute risk aversion (CARA). As underlying market impact model, we use the continuous-time liquidity model of Almgren and Chriss (2000). We show that the expected utility of sales revenues, taken over a large class of adapted strategies, is maximized by a deterministic strategy, which is explicitly given in terms of an analytic formula. The proof relies on the observation that the corresponding value function solves a degenerate HamiltonJacobi-Bellman equation with singular initial condition.
\end{abstract}

\section{Introduction}

A common problem for stock traders is to unwind large block orders of shares. These can comprise a major part of the daily traded volume of shares and create substantial impact on the asset price. The overall costs of such a liquidation can be significantly reduced by splitting the order into smaller orders that are spread over a certain time period. Thus, one possible question is to find optimal allocations for each individual placement such that the expected overall liquidity costs are minimized. Problems of this type were analyzed for various market models by Bertsimas and Lo [7], Obizhaeva and Wang [12], and Alfonsi et al. [1, 2], to mention only a few.

Taking the expected liquidity costs as a target function, however, misses the volatility risk that is associated with delaying an order. Almgren and Chriss [4, 5] therefore suggested to replace the minization of expected costs by a mean-variance optimization for sales revenues and they solved the corresponding optimization problem in the class of deterministic - or so-caled static - strategies; see also Almgren [3]. Almgren and Lorenz [6] increased the class of admissible strategies by allowing 
for intertemporal updating and found that that intertemporal updating can strictly improve mean-variance performance.

The proper economic motivation for mean-variance optimization stems from a second-order approximation of an expected utility functional as explained, e.g., in $[11$, p. 67]. We therefore propose to study directly the original problem of expectedutility maximization, and we will do this in this note for exponential, or CARA, utility functions. As an underlying market impact model we will use the same model as Almgren and Chriss [4, 5]. This simple model has the advantage that it often - and here in particular - allows for explicit computations, which makes it popular among practitioners. It also reproduces many of the key features of realworld portfolio liquidation and can display a number of highly interesting effects. We refer in particular to its applications in the analysis of economic situations with several competing traders as discussed in Brunnermeier and Pedersen [9], Carlin et al. [10], and our previous paper [15].

Our main result states that for CARA investors there is surprisingly no added utility from allowing for intertemporal updating of strategies, i.e., the expected utility is maximized by a deterministic strategy, which is explicitly given in terms of an analytic formula. In particular, the mean-variance maximizing strategy obtained in [6] will actually decrease the expected value of the exact utility.

The proof of our main result, as given in Section 3, relies on the observation that the value function of the problem solves a degenerate Hamilton-Jacobi-Bellman equation with singular initial condition, which can be solved analytically. We can thus apply verification arguments along with proper localization to deal with the singularity of the value function.

\section{Statement of main result}

We consider a large investor who needs to sell ${ }^{1}$ a position of $x>0$ shares of a risky asset by time $T>0$. The investor will thus choose a liquidation strategy that we describe by the number $X_{t}$ of shares held at time $t$ and that satisfies the boundary conditions $X_{0}=x$ and $X_{T}=0$. We assume that $t \mapsto X_{t}$ is absolutely continuous with derivative $\dot{X}_{t}$, i.e.,

$$
X_{t}=x+\int_{0}^{t} \dot{X}_{s} d s
$$

In this note, we consider one of the standard models for dealing with the price impact of such a liquidation strategy, namely the model introduced by Almgren and Chriss $[4,5]$. It is also the basis for optimal execution algorithms that are widely used in practice. In this model, the incremental order $\dot{X}_{t}$ induces a permanent price impact $\gamma \dot{X}_{t} d t$, which accummulates over time, and a temporary impact $\lambda \dot{X}_{t}$, which vanishes instantaneously and only effects the incremental order $\dot{X}_{t}$ itself. This idealization of instantaneous recovery from the temporary impact approximates reality reasonably well as long as the time intervals between the physical placement of orders are longer than a few minutes; see, e.g., Bouchaud et al. [8], Potters and Bouchaud [13], and Weber and Rosenow [16] for empirical studies on resilience in order books

\footnotetext{
${ }^{1}$ The focus on sell orders is for convenience only; symmetric statements hold for the case of buy orders.
} 
and Obizhaeva and Wang [12] and Alfonsi et al. [1, 2] for corresponding market impact models. When the large investor is not active, it is assumed that the price process $P$ follows a Bachelier model with volatility $\sigma$. The resulting stock price dynamics are hence given by

$$
P_{t}=P_{0}+\sigma B_{t}+\gamma\left(X_{t}-X_{0}\right)+\lambda \dot{X}_{t}
$$

for a standard Brownian motion $B$ starting at $B_{0}=0$ and positive constants $\sigma$, $\gamma, \lambda$, and $P_{0}$. At first sight, it might seem to be a shortcoming of this model that it allows for negative asset prices. In reality, however, a typical time span $T$ for the liquidation of a block order will be measured in days or even hours so that $P_{0} \gg \sigma \sqrt{T}$. Hence negative prices only occur with negligeable probability. Moreover, on the scale we are considering, the price process is a random walk on an equidistant lattice and thus perhaps better approximated by an arithmetic rather than, e.g., a geometric Brownian motion.

We parameterize strategies as $X_{t}=x-\int_{0}^{t} \xi_{s} d s$ with a progressively measurable process $\xi$ such that $\int_{0}^{T} \xi_{s}^{2} d s<\infty$ and $\int_{0}^{T} \xi_{s} d s=x$. We assume in addition that our strategies are admissible in the sense that the resulting position in shares, $X_{t}(\omega)$, is bounded uniformly in $t$ and $\omega$ with upper and lower bounds that may depend on the choice $\xi$. Economically, there is clearly no loss of generality in doing so as the total amount of shares available for any stock is always bounded, i.e., $X$ is always a bounded process in practice. By $\mathcal{X}(x, T)$ we denote the class of all admissible strategies $\xi$. The revenues from using such a sales strategy are given by

$$
\begin{aligned}
\mathcal{R}_{T}(\xi) & =\int_{0}^{T} \xi P_{t} d t \\
& =P_{0}\left(X_{0}-X_{T}\right)-\frac{\gamma}{2}\left(X_{T}-X_{0}\right)^{2}+\sigma \int_{0}^{T} \xi_{t} B_{t} d t-\lambda \int_{0}^{T} \xi_{t}^{2} d t \\
& =P_{0} x-\frac{\gamma}{2} x^{2}+\sigma \int_{0}^{T} X_{t} d B_{t}-\lambda \int_{0}^{T} \xi_{t}^{2} d t .
\end{aligned}
$$

Here $P_{0} x$ is the face value of the position. The term $\gamma x^{2} / 2$ corresponds to the liquidation costs resulting from the permant price impact of $\xi$. Due to the linearity of the permanent impact function, it is independent of the choice of the liquidation strategy. The stochastic integral corresponds to the volatility risk that is accummulated by selling throughout the interval $[0, T]$ rather than liquidating the portfolio instanteneously. The integral $\lambda \int_{0}^{T} \xi_{t}^{2} d t$ corresponds to the transaction costs arising from temporary market impact.

The goal of the investor is to maximize the expected utility $\mathbb{E}\left[u\left(\mathcal{R}_{T}(\xi)\right)\right]$ of the revenues when $\xi$ ranges over $\mathcal{X}(x, T)$. In this note, we consider the case in which $u$ is a CARA utility function with risk aversion $\alpha>0$, i.e.,

$$
u(x)=-e^{-\alpha x} .
$$

Let us first consider the case in which $\xi$ ranges only over the subclass $\mathcal{X}_{\operatorname{det}}(x, T)$ of deterministic strategies in $\mathcal{X}(x, T)$, i.e., strategies that do not allow for intertemporal updating. In this case, $\mathcal{R}_{T}(\xi)$ is normally distributed, and we obtain

$$
\mathbb{E}\left[u\left(\mathcal{R}_{T}(\xi)\right)\right]=-\mathbb{E}\left[e^{-\alpha \mathcal{R}_{T}(\xi)}\right]=-\exp \left(-\alpha \mathbb{E}\left[\mathcal{R}_{T}(\xi)\right]+\frac{\alpha^{2}}{2} \operatorname{var}\left(\mathcal{R}_{T}(\xi)\right)\right) .
$$


Finding the optimal liquidation strategy is now simple: it is the deterministic strategy $\xi^{*}$ that maximizes the mean-variance functional

$$
\mathbb{E}\left[\mathcal{R}_{T}(\xi)\right]-\frac{\alpha}{2} \operatorname{var}\left(\mathcal{R}_{T}(\xi)\right)
$$

over $\mathcal{X}_{\text {det }}(x, T)$; an explicit solution is given by

$$
\xi_{t}^{*}=x \sqrt{\frac{\alpha \sigma^{2}}{2 \lambda}} \cdot \frac{\cosh \left((T-t) \sqrt{\frac{\alpha \sigma^{2}}{2 \lambda}}\right)}{\sinh \left(T \sqrt{\frac{\alpha \sigma^{2}}{2 \lambda}}\right)} .
$$

This problem of mean-variance optimization has been introduced by Almgren and Chriss [4, 5] and studied extensively since; see, e.g., Almgren [3], Almgren and Lorenz [6], and the references therein. Note that that the variance is weighted here by the factor $\alpha / 2$, i.e., with half of the risk aversion parameter, in contrast to the convention in $[3,4,5,6]$ of using the full risk aversion. The formula (4) can be obtained by standard calculus of variations techniques or by passing to the limit in the corresponding discrete-time solution obtained in $[4,5]$. It is also an immediate consequence of our main theorem.

Almgren and Lorenz [6] find that allowing for dynamic updating, i.e., replacing $\mathcal{X}_{\text {det }}(x, T)$ by the entire class $\mathcal{X}(x, T)$ of admissible strategies, can improve the mean-variance performance compared to deterministic strategies. That is, (4) no longer maximizes the functional (3). For non-deterministic strategies, however, the identity (2) fails, and mean-variance optimization is no longer equivalent to the original problem of maximizing the expected utility of an investor with constant absolute risk aversion; it can only be regarded as a second-order approximation. In fact, Theorem 2.1, our main result, shows that in the original problem there is surprisingly no added utility from allowing for intertemporal updating of strategies, i.e., the deterministic strategy (4) maximizes the expected CARA utility also within the full class $\mathcal{X}(x, T)$.

Theorem 2.1 For $u(x)=-e^{-\alpha x}$ we have

$$
\max _{\xi \in \mathcal{X}(x, T)} \mathbb{E}\left[u\left(\mathcal{R}_{T}(\xi)\right)\right]=-\exp \left[-\alpha\left(P_{0} x-\frac{\gamma}{2} x^{2}\right)+x^{2} \sqrt{\frac{\lambda \alpha^{3} \sigma^{2}}{2}} \operatorname{coth}\left(T \sqrt{\frac{\alpha \sigma^{2}}{2 \lambda}}\right)\right],
$$

and the unique maximizing strategy $\xi^{*}$ is given by the deterministic function (4).

In contrast to the case of CARA utility considered in the preceding theorem, dynamic strategies can improve liquidation performance, but only in the case of a utility function with non-constant absolute risk aversion. Such varying risk aversion is clearly insufficiently captured by the mean-variance approximation, and optimal strategies are different from the deterministic strategy (4). The quantitative and qualitative properties of optimal strategies for general utility functions are subject of ongoing research; see [14]. 


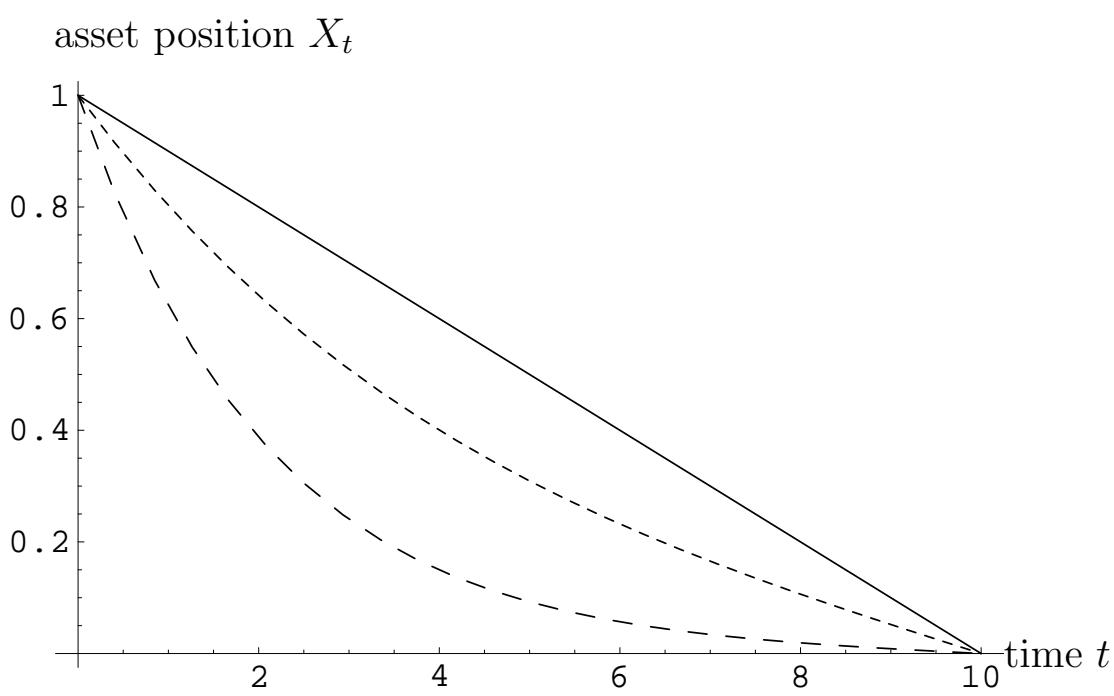

Figure 1: Asset position $X_{t}$ over time for optimal trading strategies with $x=1, T=10$, $\sigma=0.03, \lambda=0.01$. The solid line corresponds to $\alpha=0.0001$, the finely dashed line to $\alpha=1$, and the coarsly dashed line to $\alpha=5$.

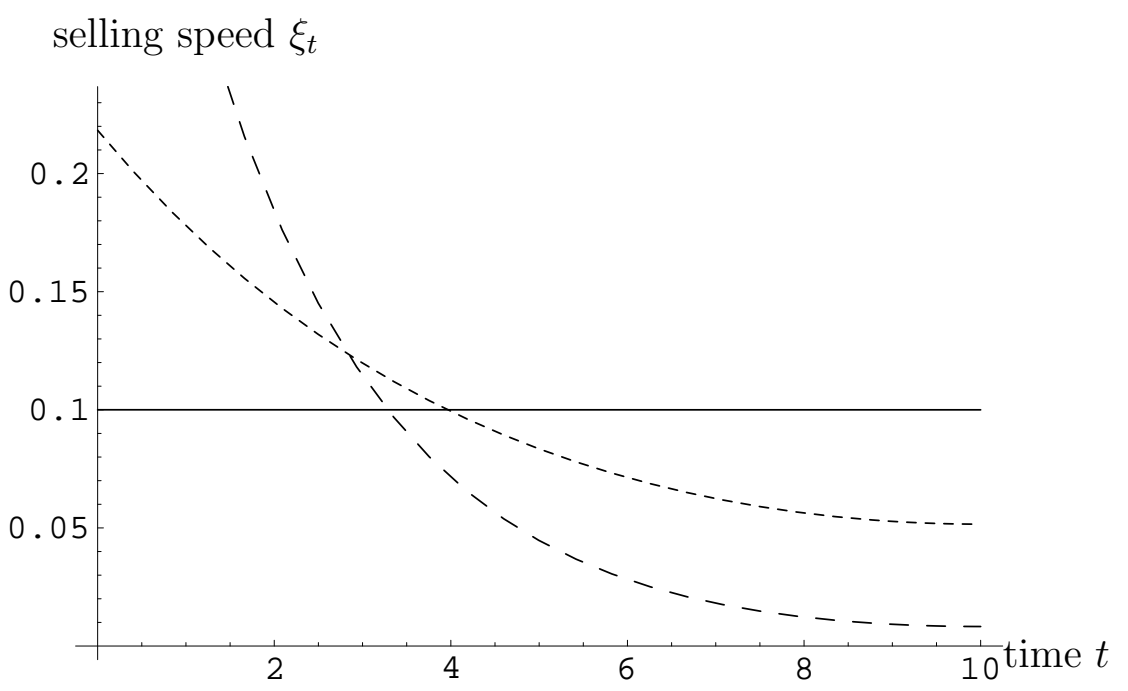

Figure 2: Selling speed $\xi_{t}$ over time for optimal trading strategies with $x=1, T=10$, $\sigma=0.03, \lambda=0.01$. The solid line corresponds to $\alpha=0.0001$, the finely dashed line to $\alpha=1$ and the coarsly dashed line to $\alpha=5$. 


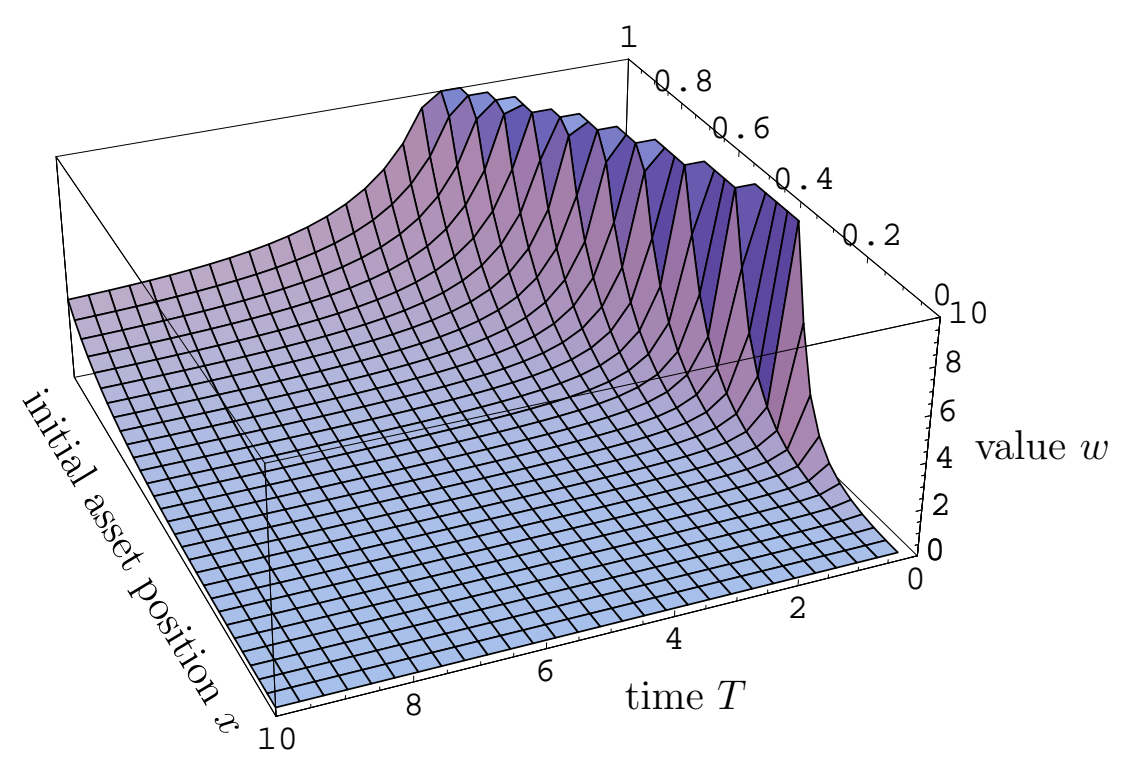

Figure 3: Value function $w(T, x, r)$ depending on the remaining time $T$ and asset position $x$ for $\alpha=1, \lambda=10, r=1$.

\section{Proof of Theorem 2.1}

Clearly, there is no loss of generality in taking $\sigma=1$. For $\xi \in \mathcal{X}(x, T)$, we define the stochastic processes

$$
X_{t}^{\xi}:=x-\int_{0}^{t} \xi_{s} d s \quad \text { and } \quad R_{t}^{\xi}:=r+\int_{0}^{t} X_{s}^{\xi} d B_{s}-\lambda \int_{0}^{t} \xi_{s}^{2} d s, \quad 0 \leq t \leq T .
$$

If we take $r=P_{0} x-\gamma x^{2} / 2$, then $R_{T}^{\xi}$ coincides with the revenue $\mathcal{R}_{T}(\xi)$ of $\xi$. But the variable $r$ can also act as an initial value of the process $R^{\xi}$ by describing revenues that carry over from the 'past' after an application of the Markov property.

In proving (5) we will simplify our formulas by dropping the first minus sign in the utility function $u(x)=-e^{-\alpha x}$. Thus, our goal is to identify the value function

$$
v(T, x, r):=\inf _{\xi \in \mathcal{X}(x, T)} \mathbb{E}\left[e^{-\alpha R_{T}^{\xi}}\right] .
$$

with the function

$$
w(T, x, r):=\exp \left[-\alpha r+x^{2} \sqrt{\frac{\lambda \alpha^{3}}{2}} \operatorname{coth}\left(T \sqrt{\frac{\alpha}{2 \lambda}}\right)\right] .
$$

To this end, we use stochastic control methods. More precisely, our proof is based on the observation that $w$ satisfies the partial differential equation

$$
w_{t}=\frac{1}{2} x^{2} w_{r r}+\frac{w_{x}^{2}}{4 \lambda w_{r}},
$$

which can be verified by a straightforward computation. Another computation shows that (7) is equivalent to the Hamilton-Jacobi-Bellman (HJB) equation

$$
w_{t}=\frac{1}{2} x^{2} w_{r r}-\sup _{\xi \in \mathbb{R}}\left(\xi^{2} \lambda w_{r}+\xi w_{x}\right) .
$$


Note that these PDEs are degenerate in the $x$-coordinate, and that $w$ satisfies a singular initial condition at $t=0$, i.e., $w(t, x, r)$ blows up for $t \downarrow 0$ for any $x>0$. This singular boundary behavior will create some technical difficulties in the proof. We define

$$
w(0,0, r):=\lim _{T \downarrow 0} w(T, 0, r)=e^{-\alpha r} .
$$

Remark 3.1 It follows from the arguments subsequent to Equation (2) that

$$
w(T, x, r)=\min _{\xi \in \mathcal{X}_{\operatorname{det}}(x, T)} \mathbb{E}\left[e^{-\alpha R_{T}^{\xi}}\right]
$$

where $\mathcal{X}_{\text {det }}(x, T)$ denotes the set of all deterministic strategies in $\mathcal{X}(x, T)$. See Remark 3.4 for an independent proof of this fact.

We start the proof of our theorem with the following a priori estimate.

Lemma 3.2 We have

$$
v(T, x, r) \geq w(T, x, r) \cdot e^{-\alpha^{2} x^{2} T / 6} .
$$

Proof: Suppose that $\xi \in \mathcal{X}(x, T)$ is such that $\mathbb{E}\left[e^{-\alpha R_{T}^{\xi}}\right]$ is finite. We then have

$$
\infty>\mathbb{E}\left[e^{-\alpha R_{T}^{\xi}}\right] \geq e^{-\alpha \mathbb{E}\left[R_{T}^{\xi}\right]} .
$$

Admissibility implies that $\mathbb{E}\left[\int_{0}^{T} X_{t}^{2} d t\right]<\infty$ and hence that

$$
\mathbb{E}\left[R_{T}^{\xi}\right]=r-\lambda \mathbb{E}\left[\int_{0}^{T} \xi_{t}^{2} d t\right] .
$$

By (10), this expression is finite, and so $\bar{x}_{t}:=\mathbb{E}\left[\xi_{t}\right]$ is well defined and belongs to $L^{1}[0, T]$, due to Fubini's theorem. Jensen's inequality implies

$$
\mathbb{E}\left[R_{T}^{\xi}\right] \leq r-\lambda \int_{0}^{T} \bar{x}_{t}^{2} d t
$$

We clearly have $\int_{0}^{T} \bar{x}_{t} d t=x$. Optimizing over all measurable functions in $L^{1}[0, T]$ that integrate up to $x$ yields

$$
\int_{0}^{T} \bar{x}_{t}^{2} d t \geq \int_{0}^{T}\left(\frac{x}{T}\right)^{2} d t=\frac{x^{2}}{T}
$$

Therefore,

$$
\mathbb{E}\left[e^{-\alpha R_{T}^{\xi}}\right] \geq e^{-\alpha \mathbb{E}\left[R_{T}^{\xi}\right]} \geq e^{-\alpha r+\alpha \lambda x^{2} / T}=: \underline{v}(x, r, T),
$$

and in turn $v(T, x, r) \geq \underline{v}(x, r, T)$.

On the other hand, the constant strategy $\xi_{t}^{0}:=x / T$ belongs to $\mathcal{X}_{\operatorname{det}}(x, T)$, and so Remark 3.1 yields that

$$
\begin{aligned}
w(T, x, r) & \leq \mathbb{E}\left[e^{-\alpha R_{T}^{\xi^{0}}}\right]=\underline{v}(x, r, T) \cdot \mathbb{E}\left[e^{-\alpha \int_{0}^{T} \frac{x(T-t)}{T} d B_{t}}\right] \\
& =\underline{v}(x, r, T) \cdot e^{\alpha^{2} x^{2} T / 6} \leq v(T, x, r) \cdot e^{\alpha^{2} x^{2} T / 6} .
\end{aligned}
$$


In the next step, we will use a verification argument to identify $w$ with the modified value function

$$
v_{0}(T, x, r):=\inf _{\xi \in \mathcal{X}_{0}(x, T)} \mathbb{E}\left[e^{-\alpha R_{T}^{\xi}}\right]
$$

that is based on the subset of $L^{2}$-bounded controls,

$$
\mathcal{X}_{0}(x, T):=\left\{\xi \in \mathcal{X}(x, T) \mid \int_{0}^{T} \xi_{t}^{2} d t \text { is bounded }\right\} .
$$

Lemma 3.3 We have $v_{0}(T, x, r)=w(T, x, r)$ and the unique minimizing strategy $\xi^{*}$ in (11) is given by the deterministic function

$$
\xi_{t}^{*}=x \sqrt{\frac{\alpha}{2 \lambda}} \cdot \frac{\cosh \left((T-t) \sqrt{\frac{\alpha}{2 \lambda}}\right)}{\sinh \left(T \sqrt{\frac{\alpha}{2 \lambda}}\right)} .
$$

Proof: The inequality $w \geq v_{0}$ is obvious from Remark 3.1. To prove the converse inequality, let $\xi \in \mathcal{X}_{0}(x, T)$ be an arbitrary control process. For $0<t<T$, Itô's formula yields that

$$
\begin{aligned}
w\left(T-t, X_{t}^{\xi}, R_{t}^{\xi}\right) & -w(T, x, r)=\int_{0}^{t} w_{r}\left(T-s, X_{s}^{\xi}, R_{s}^{\xi}\right) X_{s}^{\xi} d B_{s} \\
& -\int_{0}^{t}\left[\xi_{s}^{2} \lambda w_{r}+\xi_{s} w_{x}+w_{t}-\frac{1}{2}\left(X_{s}^{\xi}\right)^{2} w_{r r}\right]\left(T-s, X_{s}^{\xi}, R_{s}^{\xi}\right) d s .
\end{aligned}
$$

By (8), the latter integral is nonpositive, and by noting that $w_{r}=-\alpha w$ we obtain

$$
w(T, x, r) \leq w\left(T-t, X_{t}^{\xi}, R_{t}^{\xi}\right)+\alpha \int_{0}^{t} w\left(T-s, X_{s}^{\xi}, R_{s}^{\xi}\right) X_{s}^{\xi} d B_{s} .
$$

We will show next that the stochastic integral in (14) is a true martingale. To this end, observe first that

$$
\left|X_{t}^{\xi}\right|=\left|\int_{t}^{T} \xi_{s} d s\right| \leq \sqrt{(T-t) K}
$$

where $K$ is an upper bound for $\int_{0}^{T} \xi_{s}^{2} d s$. Moreover, for some constant $C_{1}$ depending on $K$ we have

$$
R_{t}^{\xi}=r+B_{t} X_{t}^{\xi}-\int_{0}^{t}\left(\xi_{s} B_{s}+\lambda \xi_{s}^{2}\right) d s \geq-C_{1}\left(1+\sup _{s \leq T}\left|B_{s}\right|\right) .
$$

Hence, for certain constants $C_{2}$ and $C_{3}$,

$$
\begin{aligned}
w\left(T-s, X_{s}^{\xi}, R_{s}^{\xi}\right) & \leq \exp \left(\alpha C_{1}\left(1+\sup _{s \leq T}\left|B_{s}\right|\right)+C_{2}(T-s) \operatorname{coth}\left((T-s) \sqrt{\frac{\alpha}{2 \lambda}}\right)\right) \\
& \leq \exp \left(\alpha C_{1}\left(1+\sup _{s \leq T}\left|B_{s}\right|\right)+C_{3}\right) .
\end{aligned}
$$

Since $\sup _{t \leq T}\left|B_{t}\right|$ has exponential moments of all orders, the martingale property of the stochastic integral in (14) follows. 
Taking expectations in (14) thus yields

$$
w(T, x, r) \leq \mathbb{E}\left[w\left(T-t, X_{t}^{\xi}, R_{t}^{\xi}\right)\right]
$$

Using the fact that

$$
v\left(T-t, X_{t}^{\xi}, R_{t}^{\xi}\right) \leq \mathbb{E}\left[e^{-\alpha R_{T}^{\xi}} \mid \mathcal{F}_{t}\right]
$$

Lemma 3.2 and (15) give

$$
w(T, x, r) \leq e^{\alpha^{2} K^{2}(T-t)^{2} / 6} \mathbb{E}\left[v\left(T-t, X_{t}^{\xi}, R_{t}^{\xi}\right)\right] \leq e^{\alpha^{2} K^{2}(T-t)^{2} / 6} \mathbb{E}\left[e^{-\alpha R_{T}^{\xi}}\right] .
$$

Sending $t \uparrow T$ and taking the infimum over $\xi \in \mathcal{X}_{0}(x, T)$ yields $w \leq v_{0}$.

To prove the formula for the optimal strategy, one first checks that the supremum in $(8)$ is attained in

$$
\widehat{\xi}(t, y)=-\frac{w_{x}(t, y, r)}{2 \lambda w_{r}(t, y, r)}=y \sqrt{\frac{\alpha}{2 \lambda}} \cdot \operatorname{coth}\left(t \sqrt{\frac{\alpha}{2 \lambda}}\right) \geq 0 .
$$

Let us define $X_{t}$ as the solution of the ODE $\dot{X}_{t}=-\widehat{\xi}\left(T-t, X_{t}\right)$ with initial condition $X_{0}=x$. Then $X_{t}=x-\int_{0}^{t} \xi_{s}^{*} d s=X_{t}^{\xi^{*}}$ for $\xi^{*}$ from (12). Note that $\xi^{*} \in \mathcal{X}_{0}(x, T)$. Thus, taking $\xi=\xi^{*}$ in (13) yields an equality in (14). Taking expectations then proves that $\xi^{*}$ is indeed an optimal strategy in $\mathcal{X}_{0}(x, T)$. Finally, since $\widehat{\xi}$ is the unique minimizer in (8), it is clear that $\xi^{*}$ is the $\mathbb{P}$-a.s. unique optimal control in $\mathcal{X}_{0}(x, T)$.

Remark 3.4 The proof of the preceding lemma can easily be modified to give an alternative proof of (9) in Remark 3.1. To this end, let $\mathcal{X}_{1}(x, T)$ denote the subset of bounded $\xi$ in $\mathcal{X}(x, T)$. Then clearly $\mathcal{X}_{\text {det }}(x, T) \subset \mathcal{X}_{1}(x, T) \subset \mathcal{X}_{0}(x, T)$. For $\xi \in \mathcal{X}_{1}(x, T)$ there exists some $K$ such that $\left|X_{t}^{\xi}\right| \leq(T-t) K$. Therefore $w(T-$ $\left.t, X_{t}^{\xi}, R_{t}^{\xi}\right) \rightarrow w\left(0,0, R_{t}^{\xi}\right)=e^{-\alpha R_{T}^{\xi}}$ as $t \uparrow T$. Using (16) and dominated convergence in (17) thus yields $w(T, x, r) \leq \mathbb{E}\left[e^{-\alpha R_{T}^{\xi}}\right]$ and in turn

$$
w(T, x, r) \leq \inf _{\xi \in \mathcal{X}_{1}(x, T)} \mathbb{E}\left[e^{-\alpha R_{T}^{\xi}}\right] \leq \inf _{\xi \in \mathcal{X}_{\operatorname{det}}(x, T)} \mathbb{E}\left[e^{-\alpha R_{T}^{\xi}}\right] \leq \mathbb{E}\left[e^{-\alpha R^{\xi_{T}^{*}}}\right]=w(T, x, r)
$$

This in particular proves (9).

Proof of Theorem 2.1: Let now $\xi \in \mathcal{X}(x, T)$ be given such that

$$
\mathbb{E}\left[e^{-\alpha R_{T}^{\xi}}\right]<\infty .
$$

We define for $k=1,2, \ldots$

$$
\tau_{k}:=\inf \left\{t \geq 0 \mid \int_{0}^{t} \xi_{s}^{2} d s \geq k\right\} \wedge T .
$$

Conditioning on $\mathcal{F}_{\tau_{k}}$ and applying Lemma 3.2 yields that

$$
\begin{aligned}
\mathbb{E}\left[e^{-\alpha R_{T}^{\xi}} ; \tau_{k}<T\right] & \geq \mathbb{E}\left[v\left(T-\tau_{k}, X_{\tau_{k}}^{\xi}, R_{\tau_{k}}^{\xi}\right) ; \tau_{k}<T\right] \\
& \geq \mathbb{E}\left[w\left(T-\tau_{k}, X_{\tau_{k}}^{\xi}, R_{\tau_{k}}^{\xi}\right) \cdot e^{-\alpha^{2}\left(X_{\tau_{k}}\right)^{2}\left(T-\tau_{k}\right) / 6} ; \tau_{k}<T\right] \\
& \geq e^{-\alpha^{2} C^{2} T / 6} \cdot \mathbb{E}\left[w\left(T-\tau_{k}, X_{\tau_{k}}^{\xi}, R_{\tau_{k}}^{\xi}\right) ; \tau_{k}<T\right] \\
& \geq 0
\end{aligned}
$$


where $C$ is a uniform upper bound for $X_{t}^{\xi}$. Since $\mathbb{E}\left[e^{-\alpha R_{T}^{\xi}} ; \tau_{k}<T\right]$ tends to zero as $k \uparrow \infty$ due to (19), we conclude that also

$$
\mathbb{E}\left[w\left(T-\tau_{k}, X_{\tau_{k}}^{\xi}, R_{\tau_{k}}^{\xi}\right) ; \tau_{k}<T\right] \longrightarrow 0 .
$$

Moreover,

$$
\mathbb{E}\left[e^{-\alpha R_{T}^{\xi}} ; \tau_{k}=T\right]=\mathbb{E}\left[w\left(0,0, R_{T}^{\xi}\right) ; \tau_{k}=T\right]=\mathbb{E}\left[w\left(T-\tau_{k}, X_{\tau_{k}}^{\xi}, R_{\tau_{k}}^{\xi}\right) ; \tau_{k}=T\right] .
$$

We may thus conclude that

$$
\begin{aligned}
& \mathbb{E}\left[e^{-\alpha R_{T}^{\xi}}\right] \\
& \quad=\mathbb{E}\left[e^{-\alpha R_{T}^{\xi}} ; \tau_{k}<T\right]+\mathbb{E}\left[e^{-\alpha R_{T}^{\xi}} ; \tau_{k}=T\right] \\
& \geq \mathbb{E}\left[w\left(T-\tau_{k}, X_{\tau_{k}}^{\xi}, R_{\tau_{k}}^{\xi}\right)\right]+\left(1-e^{-\alpha^{2} C^{2} T / 6}\right) \cdot \mathbb{E}\left[w\left(T-\tau_{k}, X_{\tau_{k}}^{\xi}, R_{\tau_{k}}^{\xi}\right) ; \tau_{k}<T\right] .
\end{aligned}
$$

Now define $\xi^{(k)}$ as $\xi$ up to time $\tau_{k}$ and for $\tau_{k}<t \leq T$ as

$$
\xi_{t}^{(k)}=X_{\tau_{k}}^{\xi} \cdot \sqrt{\frac{\alpha}{2 \lambda}} \cdot \frac{\cosh \left(\left(T-\tau_{k}-t\right) \sqrt{\frac{\alpha}{2 \lambda}}\right)}{\sinh \left(\left(T-\tau_{k}\right) \sqrt{\frac{\alpha}{2 \lambda}}\right)}
$$

Then $\xi^{(k)}$ belongs to $\mathcal{X}_{0}(x, T)$, and Lemma 3.3 yields

$$
\mathbb{E}\left[w\left(T-\tau_{k}, X_{\tau_{k}}^{\xi}, R_{\tau_{k}}^{\xi}\right)\right]=\mathbb{E}\left[e^{-\alpha R_{T}^{\xi^{(k)}}}\right] \geq w(T, x, r) .
$$

Therefore

$$
\mathbb{E}\left[e^{-\alpha R_{T}^{\xi}}\right] \geq w(T, x, r)+\left(1-e^{-\alpha^{2} C^{2} T / 6}\right) \cdot \mathbb{E}\left[w\left(T-\tau_{k}, X_{\tau_{k}}^{\xi}, R_{\tau_{k}}^{\xi}\right) ; \tau_{k}<T\right] .
$$

Using (20) now yields the result.

Acknowledgement: Support from the Deutsche Bank Quantitative Products Laboratory is gratefully acknowledged. The authors thank the Quantitative Products Group of Deutsche Bank, in particular Marcus Overhaus, Hans Bühler, Andy Ferraris, Alexander Gerko, and Chrif Youssfi for interesting discussions. This paper expresses the private opinion of the authors and does not necessarily reflect the views of Deutsche Bank.

\section{References}

[1] Alfonsi, A., Schied, A., Schulz, A. Optimal execution strategies in limit order books with general shape functions. Preprint, QP Lab and TU Berlin (2007).

[2] Alfonsi, A., Schied, A., Schulz, A. Constrained portfolio liquidation in a limit order book model. Preprint, QP Lab and TU Berlin (2007).

[3] Almgren, R. Optimal execution with nonlinear impact functions and tradingenhanced risk. Applied Mathematical Finance 10, 1-18 (2003).

[4] Almgren, R., Chriss, N. Value under liquidation. Risk, Dec. 1999. 
[5] Almgren, R., Chriss, N. Optimal execution of portfolio transactions. J. Risk 3, 5-39 (2000).

[6] Almgren, R., Lorenz, J. Adaptive arrival price. In: Algorithmic Trading III: Precision, Control, Execution, Brian R. Bruce, editor, Institutional Investor Journals (2007).

[7] Bertsimas, D., Lo, A. Optimal control of execution costs. Journal of Financial Markets, 1, 1-50 (1998).

[8] Bouchaud, J. P., Gefen, Y., Potters, M., Wyart, M. Fluctuations and response in financial markets: the subtle nature of 'random' price changes. Quantitative Finance 4, 176 (2004).

[9] Brunnermeier, M., Pedersen, L. Predatory trading. J. Finance 60, 1825-1863 (2005).

[10] Carlin, B., Lobo, M., Viswanathan, S. Episodic liquidity crises: Cooperative and predatory trading. J. Finance 65, no. 5, 2235-2274 (2007).

[11] Föllmer, H., Schied, A. Stochastic finance: an introduction in discrete time. Second edition. Berlin: de Gruyter (2004).

[12] Obizhaeva, A., Wang, J. Optimal Trading Strategy and Supply/Demand Dynamics. Forthcoming in J. Financial Markets.

[13] Potters, M., Bouchaud, J.-P. More statistical properties of order books and price impact. Physica A 324, No. 1-2, 133-140 (2003).

[14] Schied, A., Schöneborn, T. Optimal dynamic portfolio liquidation strategies. In preparation.

[15] Schöneborn, T., Schied, A. Competing players in illiquid markets: predatory trading vs. liquidity provision. Preprint, QP Lab and TU Berlin (2007).

[16] Weber, P., Rosenow, B. Order book approach to price impact. Quantitative Finance 5, no. 4, 357-364 (2005). 